\title{
Mobile Networks Unplugged
}

\author{
Jukka Manner, Marko Luoma, Jörg Ott, Jyri Hämäläinen \\ Aalto University · Department of Communication and Networking \\ PO Box 13000, 00076 Aalto, Finland \\ firstname.lastname@tkk.fi
}

\begin{abstract}
Mobile network access has seemingly become ubiquitous in industrialized countries. With data services becoming sufficiently fast, usable, and affordable, demand is growing rapidly, both in the number of users and in capacity - since most Internet/web resources continue being tailored for fast fixed line access. A lot of engineering has gone into making mobile devices appealing and sufficiently power-conserving to last for at least a day in spite of the many emerging network-driven applications. However, this device-centric view fails to recognize the "other side": the mobile access infrastructure. There, too, power consumption raises technical issues (not just) when moving towards faster mobile access and is de-facto an inhibiting factor for cellular expansion: for practical and for cost reasons. In this paper, we seek to raise awareness of the power consumption issues of mobile networks. We argue that mobile access will become, in addition to data centers and PCs, another contributing factor from the ICT sector to greenhouse gas emissions and that current mobile access technologies limit further network expansion in rural areas. Thus, we need to start working on making mobile networks much more energy-efficient than they are today, maybe even fully selfsustainable using renewable energy sources.
\end{abstract}

\section{Categories and Subject Descriptors}

C.2.2 [Network Protocols]: Applications, C.2.1 [Network Architecture and Design]: Wireless communication, C.4 [Performance of Systems]: Performance attributes reliability, availability, and serviceability.

\section{Keywords}

Energy efficiency, energy consumption, mobile networks, renewable energy, $2 \mathrm{G}, 3 \mathrm{G}$, LTE

\section{INTRODUCTION}

Information and communication services have long since become integral part of our daily lives, and the more our dependency on those and on mobile communications increases, the more the energy demand rises for providing the necessary infrastructure and powering devices. While, on the one hand, ICT helps reducing greenhouse gas emissions - directly by, e.g., substituting travel by telecooperation tools such as video conferences as well as indirectly by, e.g., improving logistics for transportation, on the other hand, offering these services impacts the environment: concerning the entire component production and distribution lifecycle all the way to (toxic) waste as well as the energy consumption for ICT operation. The latter includes data centers, PCs, and network infrastructure.

Significant progress has been made on reducing power consumption on the computation side. PCs and monitors are put to sleep mode when idle and thus reduce more than $75 \%$ of their daily energy consumption. Servers are being consolidated in datacenters where they are more efficiently run. This reduction is offset by the more power hungry generation of hardware driven by more visual and complex software, and thus increased demand for storage. The average power demand of a server has increased even with optimistic values 50 to $100 \%$ compared to servers installed in the early 2000s. And the number of servers installed has doubled or even quadrupled during the same time leading to three to eight times increased energy use compared to the beginning of millennium [1].

This has triggered a holistic analysis of datacenters to maximize the efficiency across the entire value-chain, not just individual components: from the generation of energy to its use for ICT and cooling systems to waste recycling of waste (here: dissipated heat). While current approaches include global load distribution (moving load to cold areas) and recycling for heating systems, more advanced studies consider converting dissipated heat back to electricity by using piezo elements [2].

Energy awareness has also arisen for networking equipment: [3] to reduce the electricity bill as well as reduce heat from its components (e.g., in data centers or at operators). More efficient networking technologies are emerging, such as Energy-efficient Ethernet in the IEEE 802.3az Task Force, and IP router architectures are revisited to reduce energy consumption (e.g., [4]). Yet, the fixed network infrastructure is just one element. Mobile networks, in particular since the launch of $3 \mathrm{G}$ access are already today a major consumer of electricity. With LTE, mobile operators have to prepare for even further increases in power consumption per base station. Moreover, introducing $3 \mathrm{G}$ and beyond access to cover the same area requires more and more base stations - that need to be connected to the electric grid and to the core network. Yet, given the evolution of content and services in the Internet, the growing demand for high-speed wireless access leaves operators little choice.

This motivates our work on energy-efficient and ideally selfsustaining base stations for truly wireless access: to enable maintaining and expanding the coverage of mobile networks without extending the wired infrastructure, neither the power grip nor the network infrastructure. We can identify several incentives for manufacturers and operators to explore approaches towards autonomous mobile infrastructure:

- For operators, the energy bill of current deployed mobile networks is already more than significant, surpassing the $20 \%$ of the operating costs for some of them; with LTE this will 
approach $30 \%$. Thus, means to lower the energy consumption of the wireless network are very valuable.

- National regulations may pose increased requirements for operational backup times during the power grid failures. At least in Finland, current uptimes are to be doubled over the coming years and with the increased power consumption of $3 \mathrm{G}$ and beyond platforms, this means huge investments for operators to individual base stations and communication sites.

- Maintaining or expanding network coverage in less densely populated regions requires investment into base stations and their connectivity to the core network, but also to the power grid. While base stations are available that use local power generators (burning fossil fuels), this demands additional investment and the logistics and (road) infrastructure to provide regular fuel supply. Here, difficult terrain and other conditions of the local environment may introduce further challenges and even operational vulnerabilities.

- The lack of a stable power grid [5] limits operation of (not just) mobile access networks in many countries and remote areas. Introducing mobile communications in rural areas without the need for a power grid would be of huge value to the local population. Especially this and the previous point contribute to the digital divide between industrialized and developing countries, but also between urban and rural areas that are to be overcome.

At the same time there is an EU-wide and national level incentive to reduce carbon dioxide emissions by $20 \%$ before year 2020 . From this $20 \%, 3 \%$ is expected to come from the ICT-sector. It is estimated that this amounts to more than $50 \%$ reduction of current emissions. This goal is very challenging to begin with, but with the deployment of faster wireless access and broader coverage, the challenge becomes overwhelming. With new high-speed mobile technologies using more energy than current technologies (if built with the current principles), we are facing a challenge that cannot be overlooked.

A suitable $3 \mathrm{G}$ access addressing the above issues would be built around a relatively small wired core network with an extensive wireless edge comprising only unplugged base stations, i.e., base stations not wired to the core network or the power grid. The communications use directed radio links and the power would be locally generated using renewable energy sources by means of, e.g., wind turbines, solar panels, or hydro-electric generators.

We need to consider that, in many geographical areas, we may not be able to generate enough energy using renewable sources to power a base station for any load at any given time. Thus, we need to study ways we can enable constant connectivity of some quality, e.g., we make sure phone calls always have the energy required but data services may be limited sometimes. In this paper, we seek to raise awareness of the power issues of mobile access and draft one idea towards green expansion of mobile connectivity. As background, we first provide some rough numbers about the current situation based upon which we present a concept of an unplugged wireless access network and its applicability in Section 3. We conclude the paper in Section 4.

\section{ENERGY CONSUMPTION OF MOBILE ACCESS}

We have seen a fast deployment of increasingly powerful mobile network technologies within the last decade or so. There has been evolution from the second generation $(2 \mathrm{G})\left(1^{\text {st }}\right.$ generation being the analog technologies from the 1980s up to late 1990s) mobile network technology to $3 \mathrm{G}$, and LTE is at the time of writing being deployed in at least test networks all over the world. Each new generation has brought increased speeds and more services and in the future, the mobile networks are moving to a native packetbased operation where voice is only one packet-based service among others.

With the increase of access speeds, a base station (BS) site needs more energy than the previous generation. At the same time, each new generation of mobile network technology looses in coverage to the older technology. This is illustrated in Table 2 where we have BS ranges and relative increase in required numbers of BSes for few well known mobile technologies.

It is found that required numbers of BSs are rapidly increasing with new technologies. This is mainly due to increase in carrier frequency, bandwidth and required cell throughput. On the other hand, system efficiency increase is partly compensating the undesired decay in cell range. It is noticed that a given efficiency in Table 1 is relative in nature and may vary in different environments. Moreover, we note that the same BS transmission power has been used in first four cases while in last case of Table 2 the LTE transmission power per bandwidth is increased to the same level with HSDPA.

Table 1 Assumptions for comparisons

\begin{tabular}{l|l|l|l|l|} 
& $\mathrm{f}[\mathrm{MHz}]$ & $\mathrm{W}[\mathrm{MHz}]$ & Eff & TP $[\mathrm{Mbps}]$ \\
\hline GSM 900 & 900 & 1 & 1 & 0.5 \\
\hline GSM 1800 & 1800 & 1 & 1 & 0.5 \\
\hline HSDPA & 2100 & 5 & 3 & 7.5 \\
\hline LTE & 2600 & 20 & 6 & 75.0 \\
\hline
\end{tabular}

Table 2 Ranges in dense urban and suburban environments

\begin{tabular}{l|l|l|l|l|} 
& $\mathrm{r}$ (city) & $\mathrm{r}$ (sub) & $\mathrm{N}$ (city) & $\mathrm{N}(\mathrm{sub})$ \\
\hline GSM 900 & $1.733 \mathrm{~km}$ & $3.291 \mathrm{~km}$ & 1.00 & 1.00 \\
\hline GSM 1800 & $1.043 \mathrm{~km}$ & $2.258 \mathrm{~km}$ & 2.76 & 2.12 \\
\hline HSDPA & $0.594 \mathrm{~km}$ & $1.328 \mathrm{~km}$ & 8.52 & 6.14 \\
\hline LTE & $0.284 \mathrm{~km}$ & $0.666 \mathrm{~km}$ & 37.15 & 24.42 \\
\hline LTE +6dB & $0.419 \mathrm{~km}$ & $0.980 \mathrm{~km}$ & 17.15 & 11.28 \\
\hline
\end{tabular}

As depicted in Table 2 the loss in cell range can be compensated to some extent by increasing the transmission power in BSes. Yet, as can be seen from the last two rows of Table 2, only around 1.5 times growth in LTE cell range can be achieved by 4 times higher BS transmission power. Moreover, the evolution in fixed line connections is putting a tough pressure to further increase the cell throughput in mobile systems, which will make the above problem even worse in near future. It is expected that LTE-Advanced will provide up to $1 \mathrm{Gpbs}$ on $100 \mathrm{MHz}$ bandwidth. The efficiency of LTE-Advanced will be slightly better than that of current LTE but increase will not compensate the throughput expectations that exist to this LTE version [6].

Although the numbers of Table 2 are only a rough estimate they show the inevitable development trend: forthcoming mobile network technologies require a huge increase in the number of base stations an operator must deploy, and power. While we need more hardware, each new generation of technology also requires more power to operate. A $2 \mathrm{G}$ base stations can consume around $0.5-1 \mathrm{kVA}$, but a $3 \mathrm{G}$ site already requires twice as much, $1-2 \mathrm{kVA}$, and a LTE site requires even more, around 1,5-3 kVA.

While an operator is introducing a new technology, there is an expectation that the oldest generation will be phased out. For example in Finland, GSM technology will probably be phased out over the next five years. Consequently, to offer the same level of coverage and service, an operator's energy consumption will rise 
by up to 10 times, and offering the same coverage with LTE will require up to 60 times the energy of a $2 \mathrm{G}$ network.

Furthermore, the mentioned maximum of $3 \mathrm{kVA}$ for an LTE site is only for one operator. In many deployments, there can be several operators' hardware on the same site.

\section{REMOVING THE PLUGS}

In this section we present draft an outline of an unwired mobile network that does not run on fossil fuel. We focus on the communication and network aspects, although it is likely that to reach our vision, we also need dramatic increases in the performance of current renewable energy sources.

\subsection{Overall goal}

The power consumption of a base station is a function of at least the technology (boils down to bit rate), idle power consumption of the system (and idle vs. sleep time), of the cell size (transmission power), and the activity (actual traffic). The available power is a function of the natural resources and conditions (weather for solar panels, wind for turbines). The number of customers and their expected activity leads us to a scenario with three distinct areas as depicted in Fig. 1.

The innermost (colored) region is too densely populated for unplugged stations. Such a region would typically be an urban area, where we already have the power grid, too. When we reach the energy balance threshold (white area), deployment of unplugged base stations becomes technically feasible and

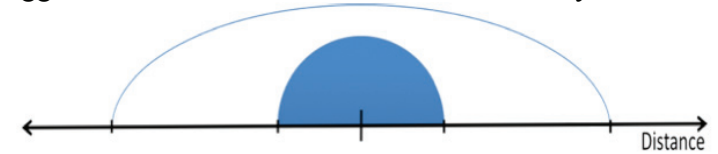

Fig. 1 Potential deployment of unplugged mobile access

economically sensible. In this area, we also have situations where the renewable energy sources do not produce enough power, and we have to use various techniques to sustain communication, at least the critical services. With further decreasing user density, we reach the sustainability border threshold, after which the system still could be operated, but insufficient return-on-investment is expected. However, even in such extreme regions, national laws may force an operator to introduce coverage.

Note that this picture changes through the seasons and the geographic location, e.g., in Finland, during winter there is little sun available compared to summer, while in southern Europe or closer to the equator, the availability of solar energy is much more stable. Similarly, wind turbines are most efficient close to shores.

Fig. 2 shows the basic concept of an unplugged mobile network. On the upper right corner we have a fixed provider core (PC) connected to other networks. This will be a wired optical network as it is today. Closer to the mobile terminals, we can have a wireless access network (AN), i.e., connecting the base stations (BS), e.g., eNB in LTE, to the PC. This can be a multi-hop wireless mesh network, or direct micro links between the PC and the base stations. We can also have BSs further out in the country that are connected to the other BSs, that eventually connect to the PC. From an architectural point of view, there is nothing new, we have had wireless mesh networks and all sorts of similar concepts out there for years - at least in research papers. Yet, most the prior work focuses on removing the communication cables but expects a power grid to be available; we don't.

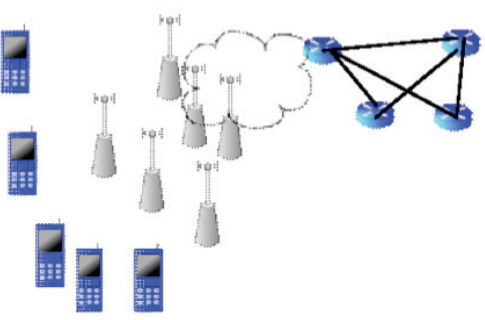

Fig. 2 An unplugged mobile network

\subsection{Challenges and Approaches}

Our concept of green, unplugged, mobile access considers only $3 \mathrm{G}$ and beyond technologies. There are already test sites [7] built for GSM service. Moreover, GSM is a technology that is in the state of phasing out in the western world in the next few years, and as discussed earlier in this paper, the power requirements of $2 \mathrm{G}$ access are vastly inferior to $3 \mathrm{G}$ and LTE.

The concept of green mobile access requires dramatic power reduction in base station power consumption. This can be tackled with several approaches that in many cases benefit from one to another. We can distinguish two categories of themes: hardware related, and network and operations related.

The following are topics on the base station hardware itself: move of the radio equipment up to the cell tower, increase of the voltage level from 48VDC to 400VDC between power unit and the radio unit, change of the power amplifier from fixed linear to envelope tracking ones, enable much lower power consumption of the wireless links, both connecting the clients and the network nodes, and design even more powerful renewable energy sources and storage capabilities.

Lifting up the radio equipment to the top of the cell tower provides also some other benefits than just lower feeder losses. It also makes units less vulnerable to illegitimate attacks but also makes maintenance harder. Free ambient cooling can be positive side effect when situated on cool territory but at extreme conditions (cold and warm) may produce difficulties in maintaining stabile environment.

Increasing the voltage levels at the base station feeding up to 400VDC makes the transmission of power much more efficient and economical as there are cheaper cables employed. Low feeding voltage (48VDC) leads to high feeding currents and either low efficiency or large cable sizes.

Application of envelope tracking is the major redactor of power usage in the base station. Envelope tracking is a power control process where knowledge of the amplitude modulated carrier waveform is used in the powering process of a power amplifier. The required control information (transmission envelope) can be obtained from the digital pre-distortion units and fed into DC/DCconverters of the power amplifier. Currently power amplifiers are operating on very low efficiency as the power is provided at the fixed level and excess is dissipated as a heat. It is commonly approximated that power amplifier uses more than $50 \%$ of the whole energy of the base station. With envelope tracking this can be reduced more than $50 \%$ [8].

Power control of wireless communications has been a popular topic for tens of years. There is nothing fundamentally different when employed to create the unplugged wireless network, besides the new application and use case. Thus, we do not discuss evolution of wireless communication and the power control of the radios in this paper, it is enough to note the theme and application. 
It is worth noting that development of powering solutions (e.g, envelope tracking) and electronics is a prerequisite for further work towards our vision. We must be able to achieve a low base power consumption for the equipment, e.g., under low load or when no users are communicating, a base station must use minimal energy in order to enable recharging the energy stores.

The same comment can be said about the renewable energy sources, our scheme benefits greatly from any advance in creating energy from renewable sources, and would enable more traffic to be handled with the power grid. Besides energy generation, storage of energy during off-peak times is also a critical task. Lead-acid batteries are heavy and rather inefficient, but e.g. regenerating fuel cells would be an interesting solution. It should be noted that we should look into storage capabilities beyond a few hours or days worth of energy, e.g., could we store energy to cover the need of a base station for up to months?

The second category of solutions is about how to run the network and include issues such as load balancing, black nodes, availability of services, and making use of weather forecasts.

The central goal in operating the network is that the operator can control and possibly even out the power consumption in the network, and avoid situations where a base station would be abruptly taken out of service due to lack of energy. Since mobile networks are a critical service to the society, an operator has strict requirements on the level and availability of the service. A prerequisite to the schemes below is accurate modeling and prediction: we need to understand exactly how much energy is consumed e.g. for voice calls, SMS and MMS messages, data transfers, and communication between network nodes, and build models and prediction algorithms that are able to give us accurate information into the future.

Balancing the load of base stations is possible when the coverage has been designed properly and we can cover any physical location with at least two base stations. Here we could move (force a handover) customers from one cell to another in order to lower the power consumption of one particular base station; currently we do the same but the goal is to maintain high quality connectivity. Naturally such a network-driven handover may result in lower quality of the connectivity and should only be one of the last options to choose.

We could also deploy so-called black nodes, base stations that are dormant and restore their energy storage, and only come alive if needed. The black nodes would come up when a given site is not getting enough power from the renewable sources to sustain the services requested by clients. Sites would thus alternate turns, one serves, the other restores.

If we cannot switch clients off the base station or take it down, we can still shut down data services with high downlink and uplink speeds. For example, we shut down HSDPA or LTE service when the power consumption grows too high compared to the power input and energy storage left. Yet, this may not be as simple because analyzes show that the energy consumption per bit of transferred data is actually better with higher speed technologies. The topic thus needs much more work to make it work right. Yet, what we can obviously do in the worst case is shutdown data service altogether and only maintain voice calls which may even be turned into push-to-talk-style or even asynchronous voice messaging to prolong inactivity times $[5,9]$.

Building an unplugged mobile network is also about weather forecasts. If the weather in the next days will be cloudy and less windy, the operator may take a conservative approach to provide mobile services in order to not let the energy storage on the base stations deplete too rapidly. On the other hand if the weather forecast indicates high winds or sunny days ahead, the energy storage could go down below some critical level because we will (might) be able to restore the storage levels in the coming days.

\section{CONCLUSIONS}

Pursuing the approach and tackling the challenges outlined above could pave the way to unplugged mobile networks in suitable areas. Their benefits would be manifold: Operators can achieve lower operational cost and make communication more affordable to the user. Reduced investment - since they are able to limit infrastructure building, maintenance, and logistics - allows operators to expand their networks more easily, serve more users, and bring (better) connectivity to rural areas, in industrialized and developing countries alike, thus making (affordable) communication more available.

But also positive macroscopic effects are expected, besides reduced energy consumption: At a national level, the benefits include lower energy consumption and less need to build new power plants. And reliability of mobile communications increases due to reduced dependency on the electric power grid with its potential vulnerabilities to weather, disasters, violence, etc.

The concepts and challenges outlined in this paper constitute only a part of all the bits and pieces that must come together to realize this vision. Yet, it would be a good start for a sustainable future for mobile communication.

\section{REFERENCES}

[1] Jonathan G. Koomey: Estimating total Power Consumption by Servers in the U.S. and the World, Final report, February 15,2007

[2] A Sound Way To Turn Heat Into Electricity, http://www.sciencedaily.com/releases/2007/06/07060322502 6.htm

[3] Maruti Gupta and Suresh Singh: Greening of the Internet. Proceedings of ACM SIGCOMM, 2003.

[4] Raffaele Bolla, Roberto Bruschi, Franco Davoli, Andrea Ranieri: Energy-Aware Performance Optimization for NextGeneration Green Network Equipment, Proc. of ACM SIGCOMM PRESTO workshop, 2009.

[5] R. J. Honicky, Omar Bakr, Michael Demmer, Eric Brewer: A message-oriented phone system for low cost connecticity. ACM HotNets 2007.

[6] 3GPP, "Requirements for Further Advancements for Evolved Terrestrial Radio Access (E-UTRA)", 3GPP Technical Report, TR 36.913 v8.0.1, March 2009.

[7] Motorola and GSM Association Deploy Wind and Solar Powered Cell Site with MTC Namibia: http://www.motorola.com/mediacenter/news/detail.jsp?global ObjectId $=8157 \quad 808623$

[8] Kaneta, M., et al., Architecture of Wideband High-Efficiency Envelope Tracking Power Amplifier for Base Station. IEICE Technical report, 2009.

[9] Md. Tarikul Islam et al.: Practical Voice Communications in Challenged Networks. ACM ExtremeCom workshop, 2009. 